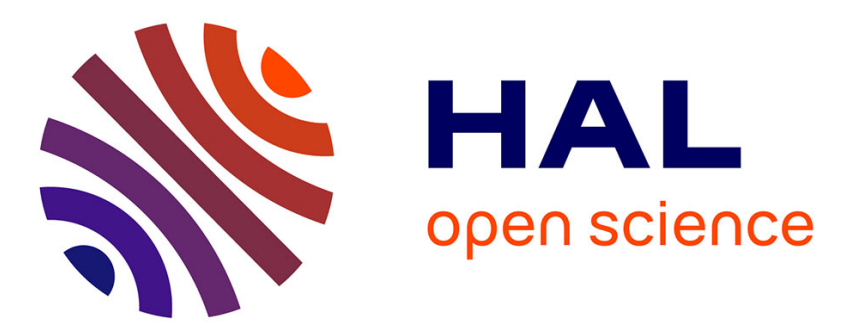

\title{
From 2D markers in MIP to 3D vessel segmentation: A fuzzy paradigm for connected filtering
}

Alice Dufour, Benoît Naegel, Christian Ronse, Joseph Baruthio, Valérie Wolff, Nicolas Passat

\section{- To cite this version:}

Alice Dufour, Benoît Naegel, Christian Ronse, Joseph Baruthio, Valérie Wolff, et al.. From 2D markers in MIP to 3D vessel segmentation: A fuzzy paradigm for connected filtering. International Symposium on Biomedical Imaging (ISBI), 2013, San Francisco, United States. pp.922-925, 10.1109/ISBI.2013.6556626 . hal-01695065

\section{HAL Id: hal-01695065 \\ https://hal.univ-reims.fr/hal-01695065}

Submitted on 3 Mar 2018

HAL is a multi-disciplinary open access archive for the deposit and dissemination of scientific research documents, whether they are published or not. The documents may come from teaching and research institutions in France or abroad, or from public or private research centers.
L'archive ouverte pluridisciplinaire HAL, est destinée au dépôt et à la diffusion de documents scientifiques de niveau recherche, publiés ou non, émanant des établissements d'enseignement et de recherche français ou étrangers, des laboratoires publics ou privés. 


\title{
FROM 2D MARKERS IN MIP TO 3D VESSEL SEGMENTATION: A FUZZY PARADIGM FOR CONNECTED FILTERING
}

\author{
A. Dufour, B. Naegel, C. Ronse, J. Baruthio ${ }^{1} \quad$ V. Wolff $^{2} \quad$ N. Passat $^{3}$ \\ ${ }^{1}$ ICube, UMR CNRS, Université de Strasbourg, France \\ ${ }^{2}$ Département de Neurologie, Hôpitaux Universitaires de Strasbourg, France \\ ${ }^{3}$ CReSTIC, EA 3804, Université de Reims, France
}

\begin{abstract}
Connected filters, and in particular those relying on component trees, can be involved in vessel filtering and segmentation tasks. We propose a new component tree-based segmentation method that enables to easily obtain vascular volumes from 3D data, by simply providing 2D markers from MIP visualisations. This approach gathers the advantages of component trees (low algorithmic cost) and the user-friendly (fuzzy) handling of 3D images via 2D representations, leading to an ergonomic and fast tool. It has been successfully used for segmenting a large dataset of MRAs and CTAs visualizing Willis polygons, in the clinical context of stenosis detection.
\end{abstract}

\section{INTRODUCTION}

Angiographic images, commonly obtained from magnetic resonance angiography (MRA) and computed tomography angiography (CTA), are useful data for several tasks, e.g., diagnosis, pathology follow-up, or surgery planning. However, due to low SNR (noise, artifacts), and complex semantic content (sparseness, venous vs. artery networks), angiographic image analysis [1] is a time consuming and error-prone task.

These considerations have motivated the development of numerous vessel filtering and segmentation techniques [2]. Among the methodological fields considered for their design, mathematical morphology has been involved for both 2D [3] and 3D [4] vessel segmentation. In this context, the approaches based on connected filtering, and especially those relying on the component tree [5], have been considered.

The component tree is a hierarchical data structure that provides a spatial and spectral model for grey-level images. It is composed by the connected components of the image, obtained at each level-set, that form the nodes of a rooted

The research leading to these results was funded by a $\mathrm{PhD}$ grant of the Région Alsace and the French Centre National de la Recherche Scientifique (CNRS). tree. The support of the image is the root of this tree, while the edges are derived from the inclusion relation between these connected components, in a set-based formalism.

From a structural point of view, the component tree is an adequate tool for representing and analyzing structures of interest that correspond to (locally) extremal intensities. This is the case for angiographic images, where the intensity maxima correspond to the flowing blood. From an algorithmic point of view, component trees can be built with a low computational cost $[5,6]$, and they also benefit from efficient node selection strategies in well-chosen frameworks $[5,7]$.

Based on these properties, several ways have been investigated to carry out vessel filtering and segmentation. In [8], Wilkinson et al. proposed an antiextensive vessel enhancement method, by removing the "noisy nodes", via (scalar) attribute selection. In [9], Caldairou et al. proposed to consider vectorial attributes for node selection, leading a segmentation method that requires a classification process to discriminate the relevant nodes in a wide parameter space. In [10], we investigated a different methodology, that no longer uses a "local" description of each node. The main idea is to come back to the definition of the component tree, and to consider -more globally- the nodes as the generators of the image. Vessel segmentation can then be performed in an example-based fashion, by selecting the set of nodes that "fit at best" a 3D vascular example (e.g., a vascular atlas [4]) which is registered on the image to be processed.

The main drawback of this last strategy is linked to the definition and mapping of such a 3D example, which remains a time-consuming task. In this article, we propose an alternative solution to the use of a 3D example. To this end, we first show (Sec. 2.1) that the energy-based formulation of the method developed in [10] can be extended to fuzzy examples/markers. We then explain (Sec. 2.2) how a 3D fuzzy marker can be conveniently generated from one (or several) 2D binary one(s), interactively defined from maximum intensity projection (MIP) visualizations. A complementary methodological discussion is provided in Sec. 3. Experimental results -obtained in a clinical context- and perspec- 
tive works, proposed in Secs. 4 and 5, conclude this article.

\section{SEGMENTATION FRAMEWORK}

\subsection{Component tree-based segmentation: a fuzzy ver- sion}

Let $I: \Omega \rightarrow V$ be a 3D angiographic image defined on the volume $\Omega$, and taking grey-level values in $V=[0, m]$. Let $\mathfrak{T}$ be the component tree of $I$, which is composed, in particular, by a set of nodes $\mathcal{N} \subseteq \mathcal{P}(\Omega)$, that is a set of subsets of $\Omega$.

Let $M \subseteq \Omega$ be a binary vascular marker defined on the support $\Omega$ of the image $I$. In the method initially proposed in [10], the vascular volume $S \subseteq \Omega$ segmented from $I$ is obtained by computing the subset $\widehat{\mathcal{N}} \subseteq \mathcal{N}$ of nodes of $\mathfrak{T}$ that "fit at best" the vascular marker $M$, which is assumed to provide a first approximation of the sought vessels.

This can be formulated as a minimization problem, that consists of solving the following equation

$$
\widehat{\mathcal{N}}=\arg \min _{\mathcal{N}^{\prime} \subseteq \mathcal{N}}\left\{d^{\alpha}\left(\bigcup_{N \in \mathcal{N}^{\prime}} N, M\right)\right\}
$$

where $d^{\alpha}$ is a distance on $\mathcal{P}(\Omega)$, defined in terms of false positives/negatives w.r.t. the marker, as

$$
d^{\alpha}(S, M)=\alpha|S \backslash M|+(1-\alpha)|M \backslash S|
$$

where $\alpha \in[0,1]$ controls the trade-off between the tolerance to false positives and false negatives.

The resolution strategy developed in [10] mainly relies on a bottom-up exploration of the nodes $\mathcal{N}$ into the component tree $\mathfrak{T}$, by considering an energy function intrinsically linked to $d^{\alpha}$ (see [10] for a whole description of the strategy).

We propose to generalize the formulation of this segmentation problem in order to now consider fuzzy markers. Indeed, by defining -without loss of correctness- a binary segmentation $S=\bigcup_{N \in \mathcal{N}^{\prime}} N \subseteq \Omega$ as a function $S$ : $\Omega \rightarrow\{0,1\}$, and no longer as a set, and the newly considered (fuzzy) marker as a grey-level image $M: E \rightarrow[0,1]$, Eq. (1) remains valid when extending $d^{\alpha}$ (Eq. (2)) into the distance $D^{\alpha}$ (on the set of functions from $\Omega$ to $[0,1]$ ) defined by

$$
D^{\alpha}(S, M)=\int_{0}^{1} d^{\alpha}\left(\lambda_{v}(S), \lambda_{v}(M)\right) \cdot d v
$$

where $\lambda_{v}$ is the thresholding operation at value $v$, defined on the functions from $\Omega$ to $[0,1]$. In particular, this continuous formulation is equivalent to the following discrete one

$$
D^{\alpha}(S, M)=\alpha \sum_{S(x)=1}(1-M)(x)+(1-\alpha) \sum_{S(x)=0} M(x)
$$

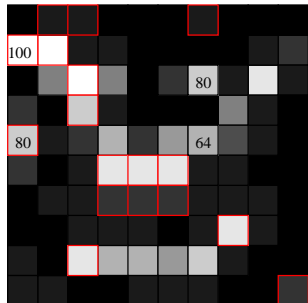

(a)

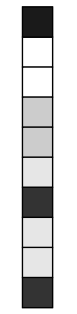

(b)

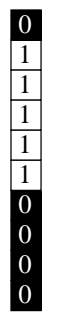

(c)

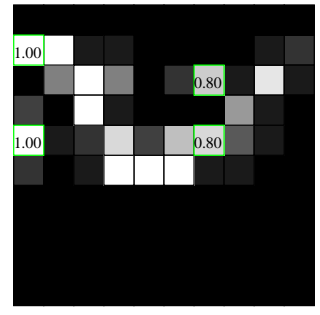

(d)
Fig. 1. (a) An image $I: \Omega \rightarrow V$. (b) The MIP image $I_{\Pi}: \Omega_{\Pi} \rightarrow V$ where $\Pi$ is the vertical hyperplane associated to the horizontal line $\mathcal{L}$. The points of $I$ which generate $I_{\Pi}$ are depicted in red in (a). (c) A binary marker. (d) The fuzzy marker induced by (c): points of different (resp. equal) values in $I$ can lead to equal (resp. different) fuzzy scores.

that authorizes an exact computation of $D^{\alpha}$. The bottom-up exploration strategy remains valid in this new fuzzy paradigm, by simply substituting $D^{\alpha}$ to $d^{\alpha}$ in the energy formulation. Moreover, from Eq. (3), the new "fuzzy" energy inherits from the additivity property of the former "binary" ones, that guarantees that the solution $\widehat{\mathcal{N}}$ can be found in linear time [7], for any $\alpha \in[0,1]$, but also that the (finite) set of solutions can be found in (quasi-)linear time for all the $\alpha \in[0,1]$.

\subsection{From 2D binary to 3D fuzzy markers}

Let $\mathcal{L}$ be a vectorial line of $\mathbb{R}^{3}$ and $\Pi$ be the vectorial (hyper)plane of $\mathbb{R}^{3}$, orthogonal to $\mathcal{L}$. The MIP image of a 3D image $I$, w.r.t. $\Pi$, is a $2 \mathrm{D}$ image $I_{\Pi}: \Omega_{\Pi} \rightarrow V$, where $\Omega_{\Pi}$ is the orthogonal projection of $\Omega$ onto $\Pi$. For any point $x \in \Omega_{\Pi}$, the value $I_{\Pi}(x)$ is defined as the maximal value of the points of $I$ projected on $x$ (see Fig. 1(a,b), for a 2D/1D example)

$$
I_{\Pi}(x)=\max \{I(y) \mid y \in(x+\mathcal{L}) \cap \Omega\}
$$

Let us consider a $2 \mathrm{D}$ binary marker $M_{\Pi}: \Omega_{\Pi} \rightarrow\{0,1\}$ defined w.r.t. $I_{\Pi}$ (see Fig. 1(c)). In particular, let us focus on a point $x \in \Omega_{\Pi}$ such that $M_{\Pi}(x)=1$, i.e., a point that is labeled as "vascular" in $I_{\Pi}$, according to $M_{\Pi}$. This crisp vascular score of 1 is directly linked to the value of $x$ in $I_{\Pi}$ and, equivalently, to the value of the point(s) $y$ within $\Omega$ satisfying Eq. (5). Such points $y$ of maximal intensity, are then assumed as vascular according to the (3D extrusion of the) marker $M_{\Pi}$.

Following that paradigm, any other points $z$ in $(x+$ $\mathcal{L}) \cap \Omega$ which have a different (actually lower) intensity than $x, y$ may be considered as having a lower vascular score, varying between 0 (for the points of null value) and 1 (for 
the points of highest value). Such fuzzy vascular scores can be conveniently obtained by defining a transfer function $\phi_{k}:[0, k] \rightarrow[0,1]$, which is monotonic, and verifies $\phi_{k}(0)=0$ and $\phi_{k}(k)=1$, where $k=I_{\Pi}(x)$, for any $x$ in $\Pi$ (functions with similar properties have been pioneered in [11]).

Based on such transfer functions, the crisp vascular hypotheses carried by the $2 \mathrm{D}$ marker $M_{\Pi}$ defined on $\Omega_{\Pi}$ can be extruded in $\Omega$, in order to provide a 3D marker $M: \Omega \rightarrow$ $[0,1]$ that carries fuzzy vascular hypotheses (see Fig. 1(d)). Practically, this marker can be defined, for any $y \in \Omega$ by

$$
M(y)=M_{\Pi}(x) \cdot \phi_{I_{\Pi}(x)}(y)
$$

where $x$ is the orthogonal projection of $y$ on $\Omega_{\Pi}$. Such 3D fuzzy markers can, in particular, be involved in the component tree-based segmentation framework considered in Sec. 2.1.

\section{METHODOLOGICAL DISCUSSION}

In this section, we propose complementary discussions in order to ease the instantiation of the general segmentation framework defined above.

Connectivity handling The definition of the (nodes of the) component tree involved in the segmentation process, relies on the chosen connectivity on $\Omega$. Beyond standard connectivities (induced by 6 - and 26 -adjacency models), alternative "vessel-oriented" ones may be considered. In particular, mask-based connectivities can be relevantly involved, in an extensive way (for vessel reconnection) [12], but also in an antiextensive one (for noise/artifact disconnection). This can be done by simply parameterising the component-tree $\mathfrak{T}$ with the chosen adjacency, before its construction.

Hierarchical segmentation map As stated in Sec. 2.1, we can compute the segmentation result $S$ associated to a given $\alpha$, but more generally, all the segmentations $S_{\alpha}$ over the whole $\alpha$-space $[0,1]$. The set of the (distinct) $S_{\alpha}$ segmentations is actually finite. Moreover, the segmentation process inherits the decreasing property from the thresholding operator. Indeed, for any $\alpha_{1}<\alpha_{2}$, we have $S_{\alpha_{2}} \subseteq S_{\alpha_{1}}$. In particular, it is possible to stack all the (binary) $S_{\alpha}$ results into a single (grey-level) $\alpha$-map, from which any segmentation $S_{\alpha}$ can be retrieved in real-time, by a thresholding over $\alpha$.

Transfer function In Sec. 2.2, the only prerequisites for the transfer functions $\phi_{k}$ are related to their boundary values and their increasingness. While a simple linear definition can be considered (see the toy-example of Fig. 1), more sophisticated ones can also be involved (e.g., exponential, polynomial, logarithmic, sigmoid). In particular, this choice can be guided by information about the formation of vascular signal, depending on the acquisition device. In this work, we have considered a linear definition. However, we have enriched this simple intensity-based profile by a supplementary spatial term. Indeed, it is possible to weight the fuzzy score of a voxel according to its distance with the voxel(s) that provide(s) the MIP value. Such a spatial term has been defined by considering Gaussian profiles centered on such "maximal" voxels, thus providing a spatial regularisation.

Multiple 2D markers The 2D binary-to-3D fuzzy marker generation, developed in Sec. 2.2, can by extended to deal with any arbitrary number of MIP visualisations and associated 2D markers. Such extensions rely either on linear or non-linear strategies. Indeed, this can be done by considering arithmetic or geometric mean values of the fuzzy maps provided by each single $2 \mathrm{D}$ marker, or by considering min or max compositions of such maps. Prior to this fusion operation, the $3 \mathrm{D}$ extrusions can also be constrained, e.g., by only considering the subspaces provided by their intersection.

\section{EXPERIMENTS AND RESULTS}

The proposed methodology has been involved in the analysis of a dataset of 43 time of flight (TOF) MRAs (Fig. 2(a)) (see [13] for acquisition parameters details) visualising the cerebral arterial network, with a specific focus on the Willis polygons. These images have been acquired for the analysis (and follow up) of acute ischemic stroke, among 48 patients younger that 45 years, with the purpose to identify monoarterial or multifocal intracranial stenoses, and the potential clinical causes of such pathological phenomena [13].

On one hand, due to the high number of images to consider in this study, a manual procedure would be hardly tractable. On the other hand, an automated segmentation would not guarantee to avoid false negatives. These considerations have motivated the use of a weakly-interactive segmentation methodology such as the proposed one.

In our experiments, the clinician user firstly provided a rough presegmentation as a $2 \mathrm{D}$ marker in a MIP visualisation (Fig. 2(b)); this -interactive- task could be performed in $15 \pm 5$ seconds, with standard interactive drawing tools. Secondly, the method computed the component-tree of the image, and processed from it and from the 3D fuzzy marker generated by the MIP 2D one, the $\alpha$-map (Fig. 2(c)) gathering all the potential segmentation results; this -automaticstep required $180 \pm 20$ seconds. Finally, the user chose a segmentation result (Fig. 2(d)) within this $\alpha$-map, by a simple thresholding; this last -interactive- task required less than 5 seconds.

In these experiments, the methodology was applied with (i) the connectivity induced by the standard 26-adjacency 


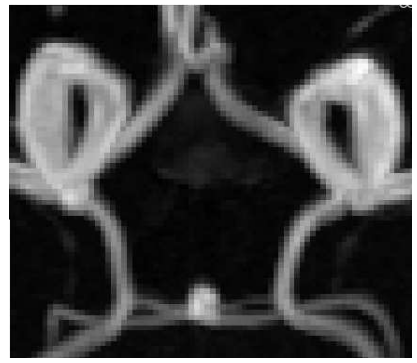

(a) MRA

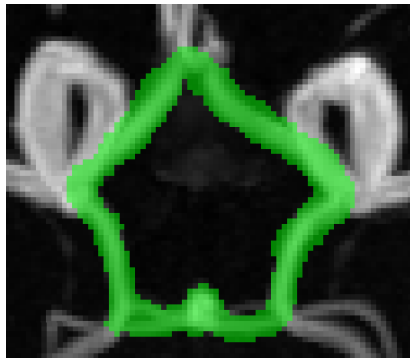

(b) Marker

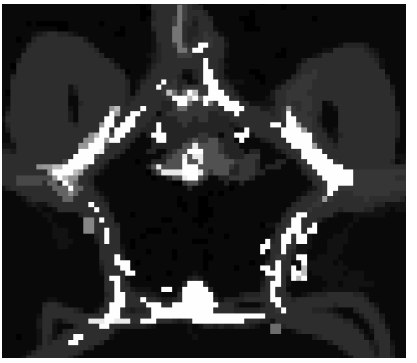

(c) $\alpha$-map

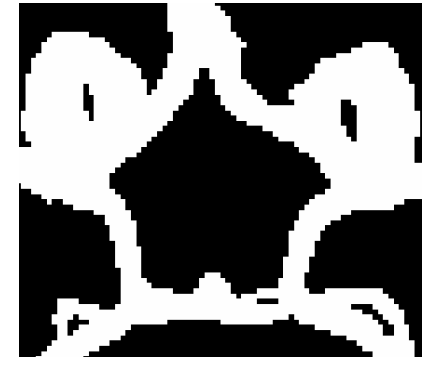

(d) Segmentation

Fig. 2. (a) TOF MRA of a Willis polygon (axial MIP visualisation). (b) 2D binary markers defined on (a). (c) Segmentation result gathered into an $\alpha$-map, automatically obtained from the component-tree segmentation of (a) w.r.t. the fuzzy marker induced from (b). (d) Binary segmentation obtained by thresholding the $\alpha$-map (c) at $\alpha=0.97$.

model; (ii) two markers defined from axial and coronal MIP visualisations with a "max" policy; and (iii) a mixed intensitylinear/spatially-Gaussian transfer function ( $\sigma=4$ voxels). The obtained segmentations, visualised by $3 \mathrm{D}$ surface rendering (Fig. 3) led to the localisation of the stenoses. In particular, the observation and handling of actual 3D vascular volumes provided to the clinician user the possibility to detect stenoses with a same accuracy, compared to MIP handling, but in a more ergonomic fashion.

\section{CONCLUSION AND PERSPECTIVES}

This work emphasises the possibility to develop user-friendly weakly interactive segmentation procedures, in the challenging context of $3 \mathrm{D}$ vessel segmentation. This has been demonstrated in the framework of mathematical morphology, that can be conveniently interfaced with fuzzy approaches.

In further works, the efficiency of the component treebased segmentation approach could be improved by also considering the connectivity as a variable that -similarly to $\alpha$ - may be tuned by the expert user. This may be considered by extending component-trees into data structures of higher dimensions (component-hypertrees) [14], and by upgrading the energy-based segmentation procedure accordingly.

\section{REFERENCES}

[1] O. Tankyevych, H. Talbot, N. Passat, M. Musacchio, and M. Lagneau, "Angiographic image analysis," in Medical Image Processing: Techniques and Applications, chapter 6, pp. 115-144. Springer, 2011.

[2] D. Lesage, E. D. Angelini, I. Bloch, and G. FunkaLea, "A review of 3D vessel lumen segmentation techniques: Models, features and extraction schemes," Medical Image Analysis, vol. 13, pp. 819-845, 2009.

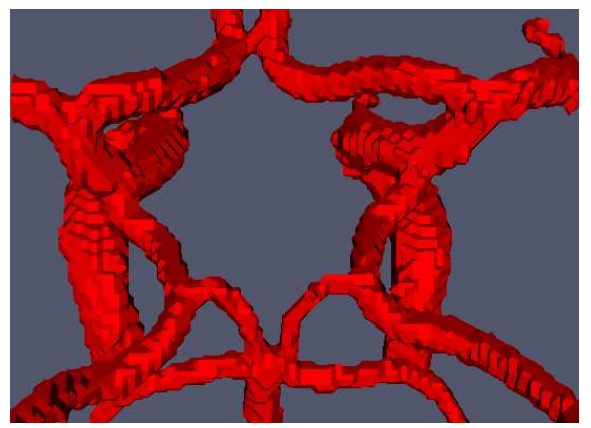

Fig. 3. 3D visualisation of the segmentation of Fig. 2(d).

[3] F. Zana and J.-C. Klein, "Segmentation of vessel-like patterns using mathematical morphology and curvature evaluation," IEEE Transactions on Image Processing, vol. 10, pp. 1010-1019, 2001.

[4] N. Passat, C. Ronse, J. Baruthio, J.-P. Armspach, and C. Maillot, "Magnetic resonance angiography: From anatomical knowledge modeling to vessel segmentation," Medical Image Analysis, vol. 10, pp. 259-274, 2006.

[5] P. Salembier, A. Oliveras, and L. Garrido, "Antiextensive connected operators for image and sequence processing," IEEE Transactions on Image Processing, vol. 7, pp. 555-570, 1998.

[6] L. Najman and M. Couprie, "Building the component tree in quasi-linear time," IEEE Transactions on Image Processing, vol. 15, pp. 3531-3539, 2006.

[7] L. Guigues, J.-P. Cocquerez, and H. Le Men, "Scalesets image analysis," International Journal of Computer Vision, vol. 68, pp. 289-317, 2006. 
[8] M. H. F. Wilkinson and M. A. Westenberg, "Shape preserving filament enhancement filtering," in $M I C$ CAI, 2001, pp. 770-777.

[9] C. Caldairou, N. Passat, and B. Naegel, "Attributefiltering and knowledge extraction for vessel segmentation," in ISVC, 2010, pp. 13-22.

[10] A. Dufour, N. Passat, B. Naegel, and J. Baruthio, "Interactive 3D brain vessel segmentation from an example," in ISBI, 2011, pp. 1121-1124.

[11] M. Vermandel, N. Betrouni, C. Taschner, C. Vasseur, and J. Rousseau, "From MIP image to MRA segmentation using fuzzy set theory," Computerized Medical Imaging and Graphics, vol. 31, pp. 128-140, 2007.

[12] O. Tankyevych, H. Talbot, P. Dokládal, and N. Passat, "Direction-adaptive grey-level morphology. Application to 3D vascular brain imaging," in ICIP, 2009, pp. 2261-2264.

[13] V. Wolff, V. Lauer, O. Rouyer, F. Sellal, N. Meyer, J. S. Raul, C. Sabourdy, F. Boujan, C. Jahn, R. Beaujeux, and C. Marescaux, "Cannabis use, ischemic stroke, and multifocal intracranial vasoconstriction," Stroke, vol. 42, pp. 1778-1780, 2011.

[14] N. Passat and B. Naegel, "Component-hypertrees for image segmentation,” in ISMM, 2011, pp. 284-295. 